\title{
Mining Unexpected Web Usage Behaviors
}

\author{
Dong (Haoyuan) $\mathrm{Li}^{1}$, Anne Laurent ${ }^{2}$, and Pascal Poncelet ${ }^{1}$ \\ ${ }^{1}$ LGI2P - École des Mines d'Alès, Parc Scientifique G. Besse, 30035 Nîmes, France \\ \{Haoyuan.Li, Pascal.Poncelet\}@ema.fr \\ ${ }^{2}$ LIRMM - Université Montpellier II, 161 rue Ada, 34392 Montpellier, France \\ laurent@lirmm.fr
}

\begin{abstract}
Recently, the applications of Web usage mining are more and more concentrated on finding valuable user behaviors from Web navigation record data, where the sequential pattern model has been well adapted. However with the growth of the explored user behaviors, the decision makers will be more and more interested in unexpected behaviors, but not only in those already confirmed. In this paper, we present our approach USER, that finds unexpected sequences and implication rules from sequential data with user defined beliefs, for mining unexpected behaviors from Web access logs. Our experiments with the belief bases constructed from explored user behaviors show that our approach is useful to extract unexpected behaviors for improving the Web site structures and user experiences.
\end{abstract}

\section{Introduction}

Recently, the applications of Web usage mining are more and more concentrated on finding valuable user behaviors from Web navigation record data (also known as Web access logs). A great deal of research work has been performed on porting data mining technologies to the Web usage analysis, in order to improve the personalization, the recommendation, and even the effectiveness of Web sites [1|2|3|4|5|6|7/8 9|10 by exploring the question: what resources are frequently visited by whom during which periods?

Among existing technologies, sequential pattern mining [11 has been well adapted to answer the above question [4/6/7/8]9]. All those sequential patterns extracted from Web access logs are typically the relationships like "on the Web site of customer support forum, $40 \%$ of users visited the TopicList page, then the Search page, then the Login page, and then the PostTopic page", or like "in the online store, $10 \%$ of customers visited the notebook cases page after having added a notebook computer to the shopping cart". This kind of relationships reflect the most general and reasonable user behaviors during Web navigations, however it become less important once we interpreted them as domain knowledge. When we regularly perform sequential pattern based Web usage mining on access logs, with the growth of the explored user behaviors, the decision makers will be more and more interested in exploring unexpected user behaviors that contradict existing knowledge, but not only in those the already confirmed.

P. Perner (Ed.): ICDM 2008, LNAI 5077, pp. 283297 2008.

(C) Springer-Verlag Berlin Heidelberg 2008 
In this paper, we focus on finding unexpected behaviors (that contradict the explored user behaviors) from Web access logs within the context of domain knowledge (that corresponds to the explored user behaviors). To illustrate our goal, let us consider an online news Web site, where the latest news are listed on the static home page index.html by categories. The latest previous news can be visited from static category index pages like cat1.html, cat2.html, etc., and all news can be visited from server side script page listnews.php by specifying the category, like listnews . php? cat $=1$ \&page $=3$. The server side script page readnews.php provides the detail of a specified news identified by news, like readnews.php?news $=20080114-002$. Assume that (1) $60 \%$ of users visit index.html, then various readnews.php, then cat1.html, then various readnews .php, then listnews . php, then various readnews . php, and then other categories and various readnews.php, etc.; (2) $10 \%$ of users visit index.html, then cat $5 . \mathrm{html}$, then various readnews . php; (3) $8 \%$ of users visit readnews . php only once; (4) $0.005 \%$ of users visit a large number of readnews . php only. From traditional sequential pattern mining approaches, we may find the most general user behaviors described in (1) with a suitable minimum support threshold, but it is quite hard to find the behaviors described in (2), (3) and (4) because:

1. Most existing sequential pattern mining approaches do not consider the missing elements, neither the semantic contradictions between elements (e.g. between cat $1 . \mathrm{html}$ and cat $5 . \mathrm{html}$ ) in a sequence. The constraint based approaches like SPIRIT 12 may find the sequences of (2) and (3), but the main drawback is that we cannot find all sequences like the one described in (2) by saying "categories contradicting cat1.html", but will have to indicate cat $2 . h t m l$, cat3.html, etc. exactly, since the constraint not cat1.html implies all pages different to cat1.html.

2. According to the model of sequential patterns, the sequences representing (2), (3) and (4) are contained in the sequences representing (1). Existing approaches that distinguish the support value of each frequent sequence (instead of maximal frequent sequence), like the closed sequential pattern [13, may find the existence of (2), (3) and (4) by computing and comparing the support values, but it is also difficult to indicate them.

The rest of this paper is organized as follows. Section 2 presents the applica-

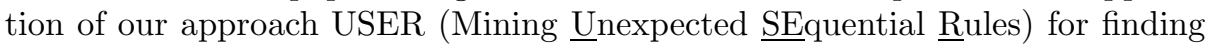
unexpected behaviors from Web access log files. In Sect. 3 we show our experimental results. We introduce the related work in Sect. 4. The conclusion is listed in Sect. 5.

\section{Finding Unexpected Behaviors from Web Access Logs}

In this section, we present the application of our approach USER for finding unexpected Web usage behaviors. We first propose a formal definition of the session sequence contained in Web access logs, then we detail our approach USER, that finds unexpected sequences and implication rules with user defined 
beliefs within the context of session sequences. We also briefly introduce the main algorithm of the approach USER.

\subsection{Session Sequences for Web Access Log Analysis}

The process of Web usage mining contains three phases, including the data preparation, pattern discovery and pattern analysis [3. The first phase is necessary for cleaning the uninterested information contained in access logs, and also for converting the log content. Then at the second phase we can apply data mining algorithms to find interesting (sequential) patterns. Finally the last phase helps the user to further analyze the results with visualization and report tools. The principle of these three phases is not different from a general data mining process.

We consider the server side access log files in the NCSA Common Logfile Format (CLF) [14, which is supported by most mainstream Web servers including the Apache HTTP Server and the Microsoft Internet Information Services. The Common Logfile Format is as follows:

remotehost rfc931 authuser [date] "request" status bytes

A $\log$ file is a ASCII text-based file, each line contains a CLF log entry that represents a request from a remote client machine to the Web server. Figure 1(a) shows the CLF log entries contained in a log file of an Apache HTTP Server. Additional fields can be combined into the CLF log entries, such as the referer field and the user agent field, shown in Fig. 1(b).

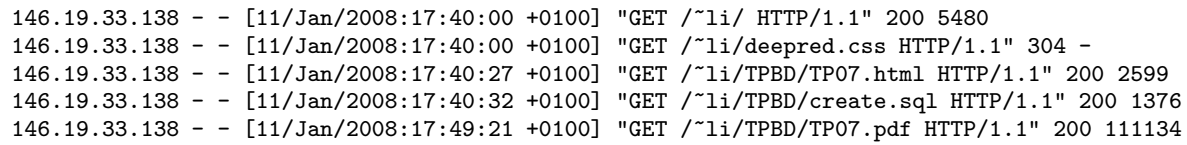

(a)

146.19.33.138 - - [11/Jan/2008:18:27:35 +0100] "GET / li/ HTTP/1.1" 2001436 "-" "Mozilla/5 .0 (Macintosh; U; Intel Mac OS X; fr-fr) AppleWebKit/523.10.6 (KHTML, like Gecko) Version/3 .0.4 Safari/523.10.6"

146.19.33.138 - - [11/Jan/2008:18:29:38 +0100] "GET / li/doc/ HTTP/1.1" 200854 "http://www lgi2p.ema.fr/ li/" "Mozilla/5.0 (Macintosh; U; Intel Mac OS X; fr-fr) AppleWebKit/523.10.6 (KHTML, like Gecko) Version/3.0.4 Safari/523.10.6"

(b)

Fig. 1. (a) Common CLF log file entries. (b) Combined CLF log file entries.

According to the definitions of item, itemset and sequence introduced in 11, an attribute is an item; an itemset $\mathcal{I}=\left(i_{1}, i_{2}, \ldots, i_{m}\right)$ is an unordered collection of items; a sequence is an ordered list $s=\left\langle\mathcal{I}_{1} \mathcal{I}_{2} \ldots \mathcal{I}_{k}\right\rangle$ of itemsets. This model is generally represented as a "Customer-Transaction-Items" relation where each sequence stands for all transactions of a customer identified by "CID" and each 
itemset stands a transaction identified by "TID". We propose the session sequences representation of Web access log entries, shown in Definition 1,

Definition 1 (Session Sequence). Let $\mathcal{L}$ be a set of Web server access log entries and $l \in \mathcal{L}$ be a log entry. A session sequence $s \sqsubseteq \mathcal{L}$ is a sequence

$$
s=\left\langle\left(i p_{s}, S_{0}^{s}\right)\left(l_{1}^{s} \cdot u r l, S_{1}^{s}\right) \ldots\left(l_{n}^{s} \cdot u r l, S_{n}^{s}\right)\right\rangle,
$$

such that for $1 \leq i \leq n, l_{i}^{s}$.url is the URL requested from IP address $i p_{s}$, and for all $1 \leq i<j \leq n$, $l_{i}^{s}$.time $<l_{j}^{s}$.time, where $l_{i}^{s}$.time and $l_{j}^{s}$.time denote the request time for log entries $l_{i}^{s}$ and $l_{j}^{s}$. $S_{0}^{s}$ is a set of items that contains all optional information of the session $s . S_{1}^{s} \ldots S_{n}^{s}$ are sets of items that contain optional information for each log entry $l_{1}^{s} \ldots l_{n}^{s}$.

The set $S_{0}^{s}$ can be empty or contain IP, date, time, user agent, and etc., for reducing the repetition of items. The sets $S_{1}^{s} \ldots S_{n}^{s}$ can be empty or contain HTTP query parameters of each access log entry. So that with session sequences, the log entries can be represented as shown in Fig. 2

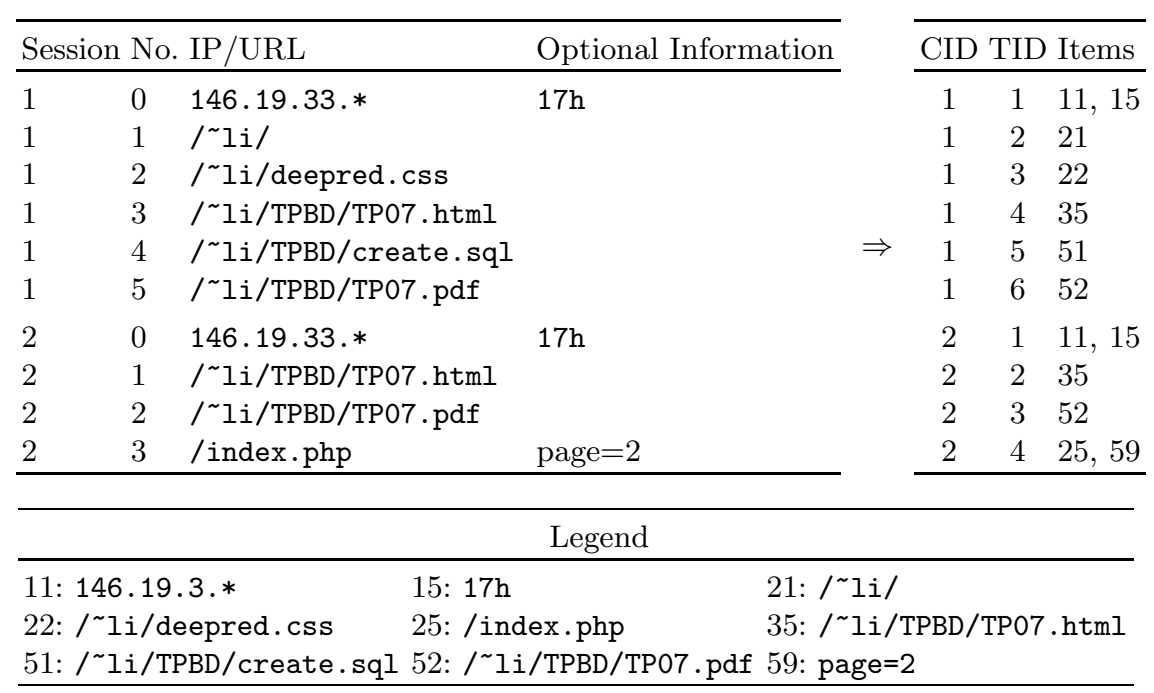

Fig. 2. Session sequence mapped CLF log entries

The sequential pattern mining finds all maximal frequent sequences with a user defined minimum support value, where the support of a sequence is defined as the fraction of the total number of sequences in the database that contain the sequence. So with the minimum support value 0.5 , the session sequences shown in Fig. 2 contain a sequential pattern $\langle(11,15)(35)(52)\rangle$, that is, $\langle(146.19 .33 . *, 17 \mathrm{~h})(\mathrm{TP} 07 . \mathrm{html})(\mathrm{TP07} . \mathrm{pdf})\rangle$. 


\subsection{Belief and Unexpectedness on Session Sequences}

Before formalizing the belief and unexpectedness on session sequences, we first introduce several additional notions, then propose the occurrence relation and implication rules between sequences.

The length of a sequence $s$ is the number of itemsets contained in the sequence, denoted as $|s|$. The concatenation of sequences is denoted as the form $s_{1} \cdot s_{2}$, so that we have $\left|s_{1} \cdot s_{2}\right|=\left|s_{1}\right|+\left|s_{2}\right|$. The notation $s \sqsubseteq^{c} s^{\prime}$ denotes that the sequence $s$ is a contiguous subsequence of the sequence $\bar{s}^{\prime}$, for example $\langle(a)(b)(c)\rangle \sqsubseteq^{c}$ $\langle(b)(\underline{a})(a, \underline{b})(\underline{c})(d)\rangle$. We denote the first itemset in a sequence $s$ as $s^{\top}$ and the last itemset as $s_{\perp}$. We therefore note $s \sqsubseteq^{\top} s^{\prime}$ if $s^{\top} \sqsubseteq s^{\prime \top}$, note $s \sqsubseteq \perp s^{\prime}$ if $s_{\perp} \sqsubseteq s^{\prime}{ }_{\perp}$, and note $s \sqsubseteq_{\perp}^{\top} s^{\prime}$ if $s^{\top} \sqsubseteq s^{\prime \top}$ and $s_{\perp} \sqsubseteq s^{\prime}{ }_{\perp}$.

Given a sequence $s$ such that $s_{1} \cdot s_{2} \sqsubseteq s$, the occurrence relation $\mapsto\langle$ op, $n\rangle$ is a constraint on the occurrences of $s_{1}$ and $s_{2}$ in $s$, where op $\in\{\neq,=, \leq, \geq\}$ and $n \in \mathbb{N}$. Let $\left|s^{\prime}\right| \models\langle\mathbf{o p}, n\rangle$ denote that the length of sequence $s^{\prime}$ satisfies the constraint $\langle\mathbf{o p}, n\rangle$, then the relation $s_{1} \mapsto\langle\mathbf{o p}, n\rangle s_{2} \operatorname{depicts} s_{1} \cdot s^{\prime} \cdot s_{2} \sqsubseteq^{c} s$ where $\left|s^{\prime}\right| \models\langle\mathbf{o p}, n\rangle$. In addition, we have $\langle\leq, 0\rangle$ implies $\langle=, 0\rangle$. We also note $s_{1} \mapsto\langle\geq, 0\rangle s_{2}$ as $s_{1} \mapsto^{*} s_{2}$, and note $s_{1} \mapsto^{\langle=, 0\rangle} s_{2}$ as $s_{1} \mapsto s_{2}$. For example, we have $\langle(a)(b)(c)\rangle \models\langle\geq, 2\rangle,\langle(a)(b)\rangle \not \models\langle>, 2\rangle,\langle(a)(b)(c)(a)(b)(c)\rangle$ satisfies $\langle(a)(b)\rangle \mapsto$ $\langle(c)\rangle$ and $\langle(a)(b)\rangle \mapsto\langle\leq, 3\rangle\langle(c)\rangle$.

We also propose the implication rule on sequences, of the form $s_{\alpha} \Rightarrow s_{\beta}$ where $s_{\alpha}$ and $s_{\beta}$ are two sequences. The rule $s_{\alpha} \Rightarrow s_{\beta}$ means that the occurrence of $s_{\alpha}$ in a sequence $s$, that is, $s_{\alpha} \sqsubseteq s$, implies $s_{\alpha} \cdot s_{\beta} \sqsubseteq^{c} s$. We constrain such a rule $s_{\alpha} \Rightarrow$ $s_{\beta}$ with the occurrence relation $s_{\alpha} \mapsto\langle\mathbf{o p}, n\rangle s_{\beta}$ between the sequences $s_{\alpha}$ and $s_{\beta}$, then the constrained rule, denoted as $s_{\alpha} \Rightarrow\langle\mathbf{o p}, n\rangle s_{\beta}$, means therefore that $s_{\alpha} \sqsubseteq s$ implies $s_{\alpha} \cdot s^{\prime} \cdot s_{\beta} \sqsubseteq^{c} s$ where $\left|s^{\prime}\right| \models\langle\mathbf{o p}, n\rangle$. Nevertheless, we further consider a semantics constraint on the rule. If the sequence $s_{\gamma}$ semantically contradicts to the sequence $s_{\beta}$, denoted as $s_{\beta} \not s_{\gamma}$, then $s_{\alpha} \sqsubseteq s$ implies $s_{\alpha} \cdot s_{\gamma} \nsubseteq^{c} s$, or implies $s_{\alpha} \cdot s^{\prime} \cdot s_{\gamma} \nsubseteq^{c} s$ with the occurrence relation constraint, where $\left|s^{\prime}\right| \models$ $\langle\mathbf{o p}, n\rangle$. With such occurrence relation and semantic contradiction constrained implication rules, we therefore define the belief on sequences as follows.

Definition 2 (Belief). A belief on sequences consists of a rule $s_{\alpha} \Rightarrow s_{\beta}$, an occurrence relation constraint $\tau=\langle\mathbf{o p}, n\rangle$, and a semantic contradiction $s_{\beta} \not s_{\gamma}$, denoted as $\left[s_{\alpha} ; s_{\beta} ; s_{\gamma} ; \tau\right]$. The rule $s_{\alpha} \Rightarrow s_{\beta}$ and the occurrence relation constraint $\tau=\langle\mathbf{o p}, n\rangle$ depict that given a sequence $s, s_{\alpha} \sqsubseteq s$ implies $s_{\alpha} \cdot s^{\prime} \cdot s_{\beta} \sqsubseteq^{c} s$, where $\left|s^{\prime}\right| \models \tau$. The semantic contradiction $s_{\beta} \nsim s_{\gamma}$ further depicts that $s_{\alpha} \sqsubseteq s$ implies $s_{\alpha} \cdot s^{\prime} \cdot s_{\gamma} \nsubseteq^{c} s$, where $\left|s^{\prime}\right| \models \tau$.

Example 1. Let us consider the online news site illustrated in Sect. 1, assume that most users visit the page index.html and then at least 3 news by the page readnews.php, and then the page cat1.html. This fact can therefore be stated by a belief with the implication rule $\langle($ index.html $)\rangle \Rightarrow$ (cat1.html) and the occurrence relation constraint $\langle\geq, 3\rangle$. If we know (by the Web site layout strategies or the previous result of sequential pattern mining) that the page cat5.html is not considered being visited two early, then we can further add the semantics constraint $\langle($ cat1.html $)\rangle \nsim\langle($ cat5.html $)\rangle$. So that finally we have the 
belief $[\langle($ index.html $)\rangle ;\langle($ cat1.html $)\rangle ;\langle($ cat $5 . h t m l)\rangle ;\langle\geq, 3\rangle]$ for describing such Web usage behaviors.

According to different beliefs, we therefore propose three forms of unexpectedness on sequences: $\alpha$-unexpectedness, $\beta$-unexpectedness and $\gamma$-unexpectedness.

Definition 3 ( $\alpha$-unexpectedness). Given a belief $b=\left[s_{\alpha} ; s_{\beta} ; s_{\gamma} ; *\right]$ and $a$ sequence $s$, if $s_{\alpha} \sqsubseteq s$ and there does not exist $s_{\beta}, s_{\gamma}$ such that $s_{\alpha} \mapsto^{*} s_{\beta} \sqsubseteq s$ or $s_{\alpha} \mapsto^{*} s_{\gamma} \sqsubseteq s$, then $s$ contains the $\alpha$-unexpectedness with respect to the belief $b$, and $s$ is so called an $\alpha$-unexpected sequence.

A belief with the occurrence relation constraint $\tau=*$ states that $s_{\beta}$ should occur after the occurrence of $s_{\alpha}$, so that a sequence $s$ violates $\tau=*$ if and only if no $s_{\beta}$ occurs in $s$ after $s_{\alpha}$. We also require that $s_{\gamma}$ should not occur after $s_{\alpha}$ in an $\alpha$ unexpected sequence, since the occurrence of $s_{\gamma}$ with respect to any constraint $\tau$ will be categorized to the $\gamma$-unexpectedness, see Definition 5 . For example, with the belief $[\langle($ index.html $)($ readnews.php $)\rangle ;\langle($ index.html $)\rangle ; \emptyset ; *]$, we can find the users who went never back to the home page index.html after reading news.

Definition 4 ( $\beta$-unexpectedness). Given a belief $b=\left[s_{\alpha} ; s_{\beta} ; s_{\gamma} ; \tau\right](\tau \neq *)$ and a sequence $s$, if $s_{\alpha} \mapsto^{*} s_{\beta} \sqsubseteq s$ and there does not exist $s^{\prime}$ such that $\left|s^{\prime}\right| \models \tau$ and $s_{\alpha} \mapsto s^{\prime} \mapsto s_{\beta} \sqsubseteq^{c} s$, then $s$ contains the $\beta$-unexpectedness with respect to belief $b$, and $s$ is so called $a \beta$-unexpected sequence.

A $\beta$-unexpectedness reflects that the implication rule is broken because the occurrence of $s_{\beta}$ violates the constraint $\tau$. For instance, as illustrated in Example 1, even as we expected that most users will visit the category index page cat $1 . \mathrm{html}$ after reading at least 3 news from the home page index.html, there exist users who read less than 3 news before leaving the home page. With analyzing the sequences containing such a $\beta$-unexpectedness stated by the belief $[\langle($ index.html $)\rangle ;\langle($ cat1.html $)\rangle ;\langle($ cat5.html $)\rangle ;\langle\geq, 3\rangle]$, we might further find that, for example, this unexpected behavior mostly happens at the moments when the site is usually less updated. So that new site promotion strategies can be positioned for these periods.

Definition 5 ( $\gamma$-unexpectedness). Given a belief $b=\left[s_{\alpha} ; s_{\beta} ; s_{\gamma} ; \tau\right]$ and $a$ sequence $s$, if $s_{\alpha} \mapsto^{*} s_{\gamma} \sqsubseteq s$ and there exists $s^{\prime}$ such that $\left|s^{\prime}\right| \models \tau$ and $s_{\alpha} \mapsto s^{\prime} \mapsto$ $s_{\gamma} \sqsubseteq^{c} s$, then $s$ contains the $\gamma$-unexpectedness with respect to belief $b$, and $s$ is so called an $\gamma$-unexpected sequence.

The $\gamma$-unexpectedness is concentrated on semantics: the occurrence of $s_{\beta}$ is replaced by its semantic contradiction $s_{\gamma}$ with respect to the constraint $\tau$. Considering again the above example, we know that the news listed on the page cat $1 . \mathrm{html}$ are semantically different to those listed on the page cat5.html (e.g. "All latest news" vs. "All old news", or "Politics" vs. "Entertainments"). If a lot of users visit the news listed on index.html then those listed on cat1.html, and only a few users visit cat5.html instead of cat1.html, then it may valuable to explore such an unexpected behavior. For example, assume that (1) from 
$08 \mathrm{~h}$ to $23 \mathrm{~h}, 60 \%$ of users confirm the explored behavior $\langle($ index.html $)\rangle \mapsto\langle\geq, 3\rangle$ $\langle($ cat $1 . \mathrm{html})\rangle$; (2) from $23 \mathrm{~h}$ to $08 \mathrm{~h}$ of the second day, $80 \%$ of users confirm the unexpected behavior $\langle($ index.html $)\rangle \mapsto\langle\geq, 3\rangle\langle($ cat5.html $)\rangle$, then it is not difficult to see that the frequency of the sequence describing the behavior (1) can be much more higher than that of those describing the behavior (2). For this reason, frequency based sequence mining approaches are difficult to extract the behavior (2), but such a behavior is valuable to decision makers.

\subsection{Unexpected Sequences and Implication Rules}

The fact that a sequence $s$ violates a given belief $b$ is denoted as $s \not \neq b$. For better describing the structure of unexpectedness, we propose the notions of the bordered unexpected sequence, the antecedent sequence and the consequent sequence within an unexpected sequence.

Definition 6 (Unexpected Sequence). A sequence $s$ that violates a belief $b=\left[s_{\alpha} ; s_{\beta} ; s_{\gamma} ; \tau\right]$ is an unexpected sequence. The bordered unexpected sequence $s_{u}$ is the maximum contiguous subsequence of $s$, (1) if $s$ is $\alpha$-unexpected, we have $s_{a} \cdot s_{u}=s\left(\left|s_{a}\right| \geq 0\right)$ such that $s_{\alpha} \sqsubseteq^{\top} s_{u}$; (2) if $s$ is $\beta$-unexpected, we have $s_{a} \cdot s_{u} \cdot s_{c}=s \quad\left(\left|s_{a}\right|,\left|s_{c}\right| \geq 0\right)$ such that $s_{\alpha} \sqsubseteq^{\top} s_{u}$ and $s_{\beta} \sqsubseteq_{\perp} s_{u}$; (3) if $s$ is $\gamma$-unexpected, we have $s_{a} \cdot s_{u} \cdot s_{c}=s\left(\left|s_{a}\right|,\left|s_{c}\right| \geq 0\right)$ such that $s_{\alpha} \sqsubseteq^{\top} s_{u}$ and $s_{\gamma} \sqsubseteq_{\perp} s_{u}$. The subsequence $s_{a}$ is the antecedent sequence. The subsequence $s_{c}$ is the consequent sequence.

Given a belief $b$ and a sequence database $\mathcal{D}$, let $\mathcal{D}_{U}$ be the set of bordered unexpected sequences of each $s \in \mathcal{D}$ that $s \not \models b, \mathcal{D}_{A}$ be the set of antecedent sequences of each $s \in \mathcal{D}$ that $s \not \models b$, and $\mathcal{D}_{C}$ be the set of consequent sequences of each $s \in \mathcal{D}$ that $s \not \models b$. We therefore define the unexpected sequential patterns and the unexpected implication rules (including the antecedent rules and the consequent rules) as follows.

Definition 7 (Unexpected Sequential Pattern). An unexpected sequential pattern $s_{U}$ is a maximal frequent sequence in $\mathcal{D}_{U}$.

The support for an unexpected sequential pattern is defined as the fraction of total number of sequences in $\mathcal{D}_{U}$ that support this unexpected sequential pattern:

$$
\operatorname{supp}\left(s_{U}\right)=\frac{\left|\left\{s \mid s_{U} \sqsubseteq s, s \in \mathcal{D}_{U}\right\}\right|}{\left|\mathcal{D}_{U}\right|} .
$$

Definition 8 (Unexpected Implication Rules). Let $u$ denote the unexpectedness stated by $b$, an antecedent rule is a rule $s_{A} \Rightarrow u$ where $s_{A}$ is a maximal frequent sequence in $\mathcal{D}_{A}$, and a consequent rule is a rule $u \Rightarrow s_{C}$ where $s_{C}$ is a maximal frequent sequence in $\mathcal{D}_{C}$.

The support for an antecedent rule $s_{A} \Rightarrow u$ (or for a consequent rule $u \Rightarrow s_{C}$ ) is defined as the fraction of total sequences in $\mathcal{D}_{A}$ (or in $\mathcal{D}_{C}$ ) that support the sequence $s_{A}$ (or the sequence $s_{C}$ ):

$$
\operatorname{supp}\left(s_{A} \Rightarrow u\right)=\frac{\left|\left\{s \mid s_{A} \sqsubseteq s, s \in \mathcal{D}_{A}\right\}\right|}{\left|\mathcal{D}_{A}\right|}
$$


or

$$
\operatorname{supp}\left(u \Rightarrow s_{C}\right)=\frac{\left|\left\{s \mid s_{C} \sqsubseteq s, s \in \mathcal{D}_{C}\right\}\right|}{\left|\mathcal{D}_{C}\right|} .
$$

In order to describe the implication relations between the antecedent/consequent sequences and the unexpectedness, we measure the confidence of the antecedent rule within the sequence database $\mathcal{D}$, and that of the consequent rule within the consequent sequence set $\mathcal{D}_{C}$ :

$$
\operatorname{conf}\left(s_{A} \Rightarrow u\right)=\frac{\left|\left\{s \mid s_{A} \sqsubseteq s, s \in \mathcal{D}_{A}\right\}\right|}{\left|\left\{s \mid s_{A} \sqsubseteq s, s \in \mathcal{D}\right\}\right|}
$$

and

$$
\operatorname{conf}\left(u \Rightarrow s_{C}\right)=\frac{\left|\left\{s \mid s_{C} \sqsubseteq s, s \in \mathcal{D}_{C}\right\}\right|}{\left|\left\{\mathcal{D}_{C}\right\}\right|} .
$$

The unexpected sequential patterns reflect the internal structure of the unexpectedness, and the unexpected implication rules reflect the implications and influences of the unexpectedness. For instance, as illustrated in the precedent example for describing Definition 5, assume that from $23 \mathrm{~h}$ to $08 \mathrm{~h}$ of the second day, $80 \%$ of users confirm the unexpected behavior $\langle($ index.html $)\rangle \mapsto\langle\geq, 3\rangle$ $\langle($ cat5.html $)\rangle$, then an antecedent rule can be:

$$
\langle(23 \mathrm{~h}-08 \mathrm{~h})\rangle \Rightarrow\langle(\text { index.html })\rangle \not\langle\langle\geq, 3\rangle\langle(\text { cat5.html })\rangle,
$$

and the confidence of such a rule is 0.8 . Assume that $60 \%$ of users who violate the behavior $\langle($ index.html $)\rangle \mapsto\langle\geq, 3\rangle\langle($ cat5.html $)\rangle$ like to click visit the advertisement displayed by ads3.php, then a consequent rule can be:

$$
\langle(\text { index.html })\rangle \not \leftrightarrow\langle\geq, 3\rangle\langle(\text { cat5.html })\rangle \Rightarrow\langle(\operatorname{ads} 3 . \mathrm{php})\rangle .
$$

If we further assume that a unexpected sequential pattern within the unexpectedness $\langle($ index.html $)\rangle \not\langle\langle\geq, 3\rangle\langle($ cat5.html $)\rangle$ is explored with support value 0.9 , for example, the sequential pattern $\langle($ index.html)(readnews.php)(cat3.html) $\rangle$, then all those facts can interpreted as following:

"Between 23h and 8h, 80\% of users do not follow the explored behavior, 90\% of those users read news listed on the home page, and then visit the category 3 instead of visiting the category 5, and then $60 \%$ of them follow the same kind of online advertisements."

Such unexpected user behaviors can be enough important to decision makers for pushing new Web site design or collaboration strategies.

\subsection{The Algorithm USER}

Figure 3 briefly shows the algorithm USER. The algorithm accepts a sequence database $\mathcal{D}$, a user defined belief base $\mathcal{B}$, a minimum support threshold min_supp and a minimum confidence threshold min_conf as inputs. It finds all unexpected sequences contained in the sequence database $\mathcal{D}$ with respect to each unexpectedness $u$ stated by each belief $b \in \mathcal{B}$. If a sequence $s$ is unexpected to $b$, then 
$s$ will be partitioned to the antecedent sequence $s_{a}\left(\left|s_{a}\right| \geq 0\right)$, the unexpected bordered sequence $s_{u}$ and the consequent sequence $s_{c}\left(\left|s_{c}\right| \geq 0\right)$. When all unexpected sequences have been extracted, the algorithm starts finding unexpected sequential patterns from each group of unexpected bordered sequences with the minimum support threshold min_supp. Finally the algorithm generates the antecedent/consequent rules from each group of antecedent/consequent sequences with the minimum confidence threshold min_conf.

A detailed description of the algorithm USER is listed in [15.

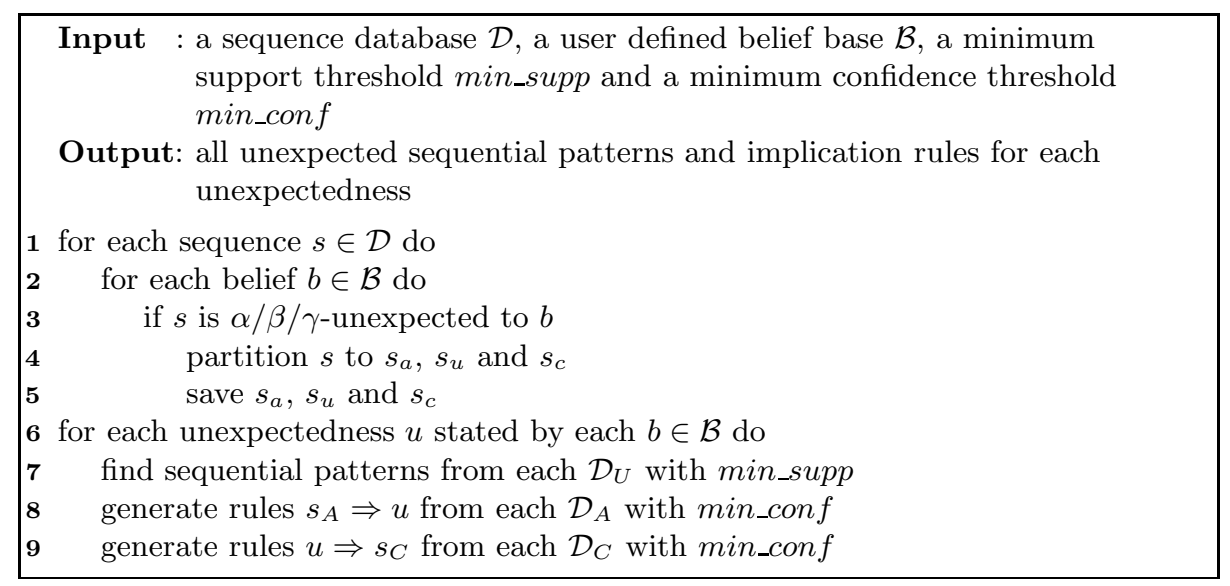

Fig. 3. Sketch of the algorithm USER

\section{$3 \quad$ Experiments}

To evaluate of our approach, we performed a number of experiments on two large access log files containing the access records of two Web servers during a period of 3 months. The first log file, labeled as LOGBBS, corresponds to a PHP based discussion forum Web site of an online game provider; the second log file, labeled as LOGWWW, corresponds to a laboratory Web site that also hosts the personal home pages of researchers and teaching staffs.

In our experiments, we split each log file into three 1-month period files, i.e., LOGBBS $-\{1,2,3\}$ and LOGWWW- $\{1,2,3\}$. We generate the session sequences with the information of day (Monday to Sunday) and hour (0h to $23 \mathrm{~h}$ ) of the first $\log$ entry of a session. If the interval time of two log entries with the same remote client IP address is greater than 30 minutes, then the last log entry starts a new session sequence. Because the CLF log entry does not contain the "remoteport" information, at this moment we do not identify the accesses from remote clients hidden behind the proxy servers and NAT gateways, so that long session sequences with the same remote address will be cut into multiple sequences with a length no more longer than 50 . 
For each session sequence, the remote client IP address is considered as a block, such that the IP 146.19.33.138 will be converted to 146.19.33.*. We map only significant HTTP query parameters to items. For LOGBBS, the number of PHP page are very limited and the parameter can stand for an access request. For example, the request

\section{/forumdisplay $\cdot p h p ? f=2 \&$ sid=f2efeb85f cfd94ecbc2dba0f97b678a1}

can be considered as an itemset $(f=2)$, and the request

/viewtopic $\cdot$ php?t=57\&sid=f2efeb85f cfd94ecbc2dba0f97b678a 1

can be replaced by the itemset $(t=57)$. We focus on the accesses of static HTML pages, server side script pages and JavaScript scripts, hence all other unconcerned files like cascading style sheets, images and data files are ignored (PDF and PS files are kept for LOGWWW). Table1 details the number of session sequences and distinct items, and the average length of the session sequences contained in the Web access logs.

Table 1. Web access logs in our experiments

\begin{tabular}{lrrr}
\hline Access Log & Sessions & Distinct Items & Average Length \\
\hline LOGBBS-1 & 27,294 & 38,678 & 12.8934 \\
LOGBBS-2 & 47,868 & 42,052 & 20.3905 \\
LOGBBS-3 & 28,146 & 33,890 & 8.5762 \\
LOGWWW-1 & 6,534 & 8,436 & 6.3276 \\
LOGWWW-2 & 11,304 & 49,242 & 7.3905 \\
LOGWWW-3 & 28,400 & 50,312 & 9.5762 \\
\hline
\end{tabular}

In order to compare our approach with the sequential pattern mining, we first apply the sequential pattern mining algorithm to find the frequent behaviors from LOGBBS $-\{1,2,3\}$ and LOGWWW- $\{1,2,3\}$ with different minimum support thresholds, shown in Fig. 4 and Fig. 5. In an acceptable range, the number of sequential patterns discovered are similar in all of the 3 periods, that increases the difficulty in analyzing new user behaviors. The post analysis shows that, for instance, within the frequent behaviors discovered with the minimum support 0.04 from LOGBBS- $\{1,2,3\}, 149$ sequential patterns discovered from all of LOGBBS $-\{1,2,3\}$ are similar (contained in each other), i.e. $>40 \%$ of LOGBBS -1 ; 197 sequential patterns discovered from LOGBBS $-\{2,3\}$ are similar, i.e., the similarity of accesses is $>70 \%$ in these two periods.

We then construct the belief bases from the workflow and those frequent behaviors discovered by sequential pattern mining. For LOGBBS, we first generate 5 beliefs from the workflow considered on this forum site, and then generate 5 beliefs from a set of selected sequential patterns discovered from LOGBBS-1. The following belief corresponds to an "expected" forum browsing order:

$$
[\langle(/)\rangle ;\langle(\mathrm{t}=2)(\mathrm{t}=5)\rangle ;\langle(\mathrm{t}=5)(\mathrm{t}=2)\rangle ;\langle=, 0\rangle]
$$




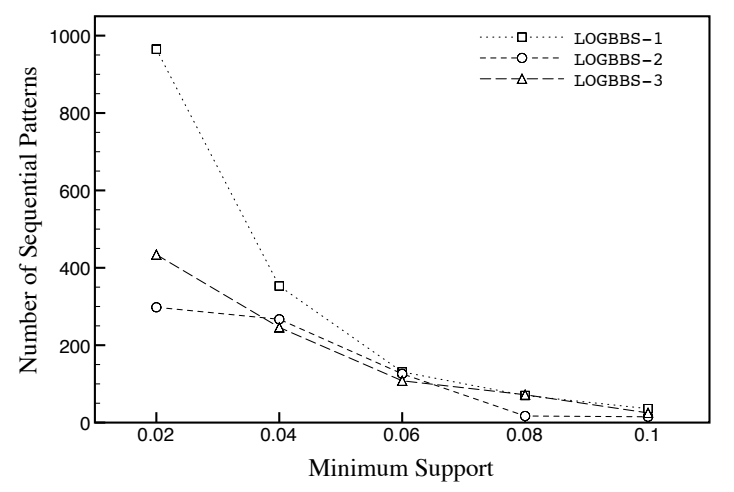

Fig. 4. Frequent behaviors discovered by the sequential pattern mining algorithm from LOGBBS

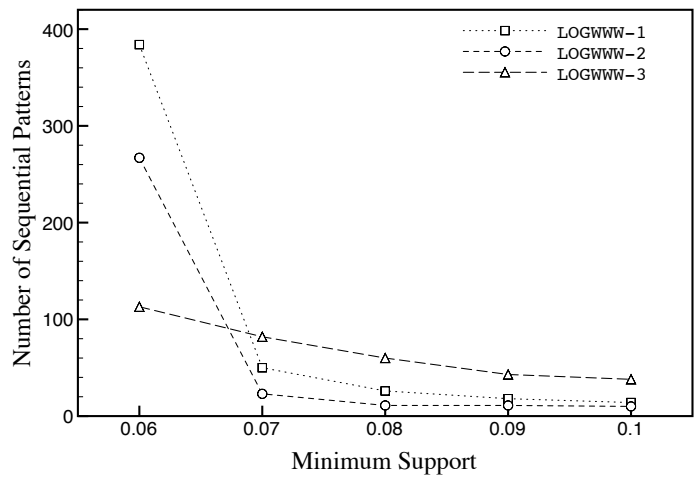

Fig. 5. Frequent behaviors discovered by the sequential pattern mining algorithm from LOGWWW

For LOGWWW we create 10 beliefs corresponding to the most frequent behaviors discovered from LOGWWW-1, for example, according to the navigation menu on the home page of this Web server, we have:

$$
[\langle(0018-04 . \mathrm{html})\rangle ;\langle(0019-27 . \mathrm{html})\rangle ;\langle((0018-04 . \mathrm{html})\rangle ;\langle\leq, 5\rangle],
$$

where 0018-04.html corresponds to the index page of the section "Research", 0019-27.html corresponds to a subsection in "Research" and 0018-04.html corresponds to a subsection in the section "Publications".

Figure 6 and Fig. 7 show the number of unexpected sequential rules discovered by our approach USER. With comparison between the quantities of frequent user behaviors, our approach generates less than the sequential pattern mining approaches. The analysis of similarity shows that, with the minimum confidence 0.2 , only 3 rules are similar from LOGBBS- $\{1,2,3\}$, 4 similar rules from LOGBBS- $\{1,3\}$, and from LOGWWW- $\{2,3\}$ we find only 1 similar rule. 


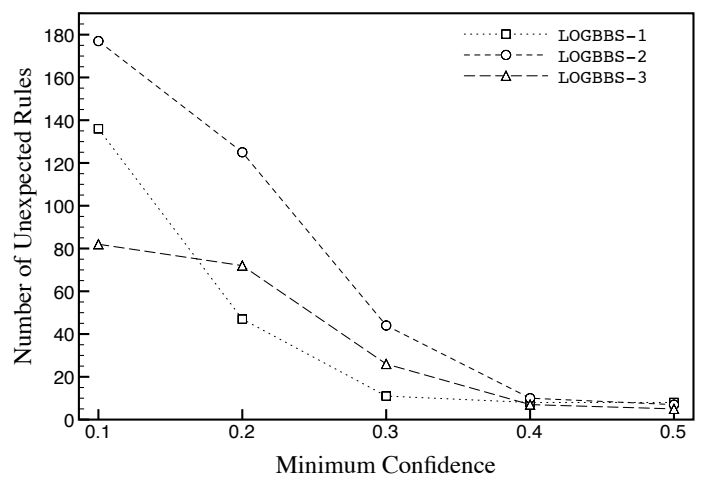

Fig. 6. Unexpected implication rules discovered by the approach USER from LOGBBS

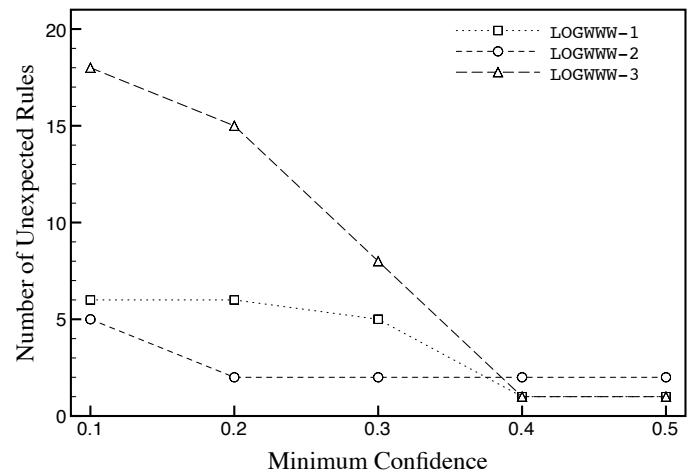

Fig. 7. Unexpected implication rules discovered by the approach USER from LOGWWW

We add 10 unexpected rules discovered from LOGBBS-1/LOGWWW-1 into the belief base for LOGBBS-2/LOGWWW-2, and add 10 (or all of them if the number is less than 10) unexpected rules discovered from LOGBBS- $\{1,2\} / \operatorname{LOGWW}-\{1,2\}$ into the belief base for LOGBBS-3/LOGWWW-3, where these latest discovered unexpected rules are considered as explored behaviors. Table 211 shows the results.

The principle of our approach does not imply that to add latest-discovered unexpected behaviors into the belief base will regularly affect the number of unexpected rules discovered from the next-period data, however, Table 2 also shows that the number of unexpected implication rules increased between LOGWWW-1 and LOGWWW- $\{2,3\}$, that is because the Web server corresponding to LOGWWW contains a great deal of non-profilable (e.g. personal home pages) Web content and the accesses are highly depended on the period, so that the beliefs could not be coherent to all data contained in LOGWWW.

\footnotetext{
${ }^{1}$ Number of beliefs: Number of unexpected implication rules.
} 
Table 2. Unexpected rules with the increment of explored behaviors

\begin{tabular}{cllllll}
\hline min_conf & LOGBBS-1 & LOGBBS-2 & LOGBBS-3 & LOGWWW-1 & LOGWWW-2 & LOGWWW-3 \\
\hline 0.1 & $10: 136$ & $20: 24$ & $30: 127$ & $10: 6$ & $16: 42$ & $26: 34$ \\
0.2 & $10: 47$ & $20: 18$ & $30: 19$ & $10: 6$ & $16: 18$ & $26: 25$ \\
0.3 & $10: 11$ & $20: 16$ & $30: 12$ & $10: 5$ & $15: 9$ & $24: 12$ \\
0.4 & $10: 8$ & $18: 9$ & $27: 4$ & $10: 1$ & $11: 9$ & $20: 10$ \\
0.5 & $10: 8$ & $18: 6$ & $24: 4$ & $10: 1$ & $11: 8$ & $19: 10$ \\
\hline
\end{tabular}

\section{Related Work}

In application domain, a great deal of Web analyzing tools, like the Webalizer [16], offer statistics based Web access analysis. In research domain, many Web usage mining approaches focus on the personalization and recommendation of Web sites by finding the most frequent user behaviors. To the best of our knowledge, we propose the first approach to unexpected Web usage mining, in considering constraints on both of the occurrence and semantics. Our approach considers a knowledge based subjective interestingness measure on sequence mining. As being summarized in [17, the interestingness measures for data mining can be classified as objective measures and subjective measures. Objective measures typically depend on the structure of extracted patterns and the criteria based on the approaches of probability and statistics (e.g. support and confidence); subjective measures are generally user and knowledge oriented, the criteria can be actionability, unexpectedness etc.. The belief driven unexpectedness is first introduced by [18 as a subjective measure where beliefs are categorized to hard beliefs and soft beliefs. A hard belief is a constraint that cannot be changed with new evidences, and any contradiction of a hard belief implies the error in gathering new evidence. A soft belief is a constraint that can be changed with new evidences by updating the degree of belief, and the interestingness of new evidence is measured by the changes of degree of belief.

Based on the proposition of [18, in the most recent approach to unexpected association rule mining presented by [19]. The mining process is done by the APriori based algorithms that find the minimal set of unexpected association rules with respect to a set of user defined beliefs. On sequence mining, 20] proposed a framework for finding unexpected sequential rules based on the frequency. In this approach, the author defines the unexpectedness from the constraints on sequential rules that depict the frequency of the content contained in a discovered sequential pattern, and the goal is to find all the sequences that do not satisfy given statistical frequency constraints.

\section{Conclusion}

In this paper we present the application of our general purposed approach USER for mining unexpected Web usage behaviors. We propose the formal definition of 
session sequence contained in Web access log files, for mining user behaviors. We introduce the notions of belief and unexpectedness within the context of session sequences, and propose the unexpected sequential patterns and implication rules. With finding unexpected implication rules, unexpected user behaviors can be studied in order to improve Web site structures and user experiences.

We also present our experiments on the access log files form different kinds of Web sites, the preprocess of such access logs and the management of beliefs are also introduced. Our experimental results show that the effects of unexpected Web usage mining highly depend on explored user behaviors, purpose, and structure of a Web site.

We are interested in applying similarity and fuzzy related approaches to unexpected Web usage mining, such as "many users like to visit the pages similar to some.page at about $6 \mathrm{~h}$ p.m.". We are also interested in mining unexpected user behaviors with hierarchical data, for example, with the categories of Web pages, or with the path information contained in the URLs, in order to find more pertinent rules.

\section{References}

1. Büchner, A.G., Mulvenna, M.D.: Discovering internet marketing intelligence through online analytical web usage mining. SIGMOD Record 27(4), 54-61 (1998)

2. Spiliopoulou, M., Pohle, C., Faulstich, L.: Improving the effectiveness of a web site with web usage mining. In: WEBKDD, pp. 142-162 (1999)

3. Srivastava, J., Cooley, R., Deshpande, M., Tan, P.-N.: Web usage mining: Discovery and applications of usage patterns from web data. SIGKDD Explorations 1(2), 12$23(2000)$

4. Mobasher, B., Dai, H., Luo, T., Nakagawa, M.: Using sequential and non-sequential patterns in predictive web usage mining tasks. In: ICDM, pp. 669-672 (2002)

5. Eirinaki, M., Vazirgiannis, M.: Web mining for web personalization. ACM Trans. Internet Techn. 3(1), 1-27 (2003)

6. Masseglia, F., Teisseire, M., Poncelet, P.: HDM: A client/server/engine architecture for real-time web usage mining. Knowl. Inf. Syst. 5(4), 439-465 (2003)

7. Huang, Y.-M., Kuo, Y.-H., Chen, J.-N., Jeng, Y.-L.: NP-miner: A real-time recommendation algorithm by using web usage mining. Knowl.-Based Syst. 19(4), 272-286 (2006)

8. Missaoui, R., Valtchev, P., Djeraba, C., Adda, M.: Toward recommendation based on ontology-powered web-usage mining. IEEE Internet Computing 11(4), 45-52 (2007)

9. Masseglia, F., Poncelet, P., Teisseire, M., Marascu, A.: Web usage mining: Extracting unexpected periods from web logs. In: DMKD (2007)

10. Mobasher, B.: Data mining for web personalization. In: The Adaptive Web, pp. 90-135 (2007)

11. Agrawal, R., Srikant, R.: Mining sequential patterns. In: ICDE, pp. 3-14 (1995)

12. Garofalakis, M.N., Rastogi, R., Shim, K.: SPIRIT: Sequential pattern mining with regular expression constraints. In: VLDB, pp. 223-234 (1999)

13. Yan, X., Han, J., Afshar, R.: CloSpan: Mining closed sequential patterns in large databases. In: SDM (2003) 
14. NCSA HTTPd Development Team: NCSA HTTPd Online Document: TransferLog Directive (1995), http://hoohoo.ncsa.uiuc.edu/docs/setup/httpd/TransferLog.html

15. Li, D.H., Laurent, A., Poncelet, P.: Mining unexpected sequential patterns and rules. Technical Report RR-07027 (2007), Laboratoire d'Informatique de Robotique et de Microélectronique de Montpellier (2007)

16. Barrett, B.L.: Webalizer (1997-2006), http://www.mrunix.net/webalizer/

17. McGarry, K.: A survey of interestingness measures for knowledge discovery. Knowl. Eng. Rev. 20(1), 39-61 (2005)

18. Silberschatz, A., Tuzhilin, A.: On subjective measures of interestingness in knowledge discovery. In: KDD, pp. 275-281 (1995)

19. Padmanabhan, B., Tuzhilin, A.: On characterization and discovery of minimal unexpected patterns in rule discovery. IEEE Trans. Knowl. Data Eng. 18(2), 202$216(2006)$

20. Spiliopoulou, M.: Managing interesting rules in sequence mining. In: Żytkow, J.M., Rauch, J. (eds.) PKDD 1999. LNCS (LNAI), vol. 1704, pp. 554-560. Springer, Heidelberg (1999) 